Note

\title{
Detection Method for Aquatic Bacteria of the Fingers, as a Potential Origin of the Aqueous Solution Contamination
}

\author{
EIICHI OSONO ${ }^{1,2 *}$, KAZUMI HONDA $^{1}$, YUKI INOUE ${ }^{1}$, YOSHIHIKO NOROSE ${ }^{2}$, \\ MEGUMI TAKAHASHI ${ }^{2}$, KYOKO ICHIMURA', CHISAKO KAMANO', EIJI SHINYA ${ }^{2}$, \\ SHUN TAKAKU ${ }^{2}$, KENTARO OKAMATSU ${ }^{3}$, SHINYA KAWAMOTO ${ }^{4}$, HIDEAKI TAKIZAWA $^{5}$, \\ AND HIDEMI TAKAHASHI \\ ${ }^{1}$ Koshigaya Ohbukuro Clinic. 1557-25 Fukuroyama, Koshigaya, Saitama 343-0032, Japan \\ ${ }^{2}$ Microbiology and Immunology, Nippon Medical School. 1-25-16 Nezu, Bunkyo, Tokyo 113-0031, Japan \\ ${ }^{3}$ Komagome Aoba Clinic. 1-38-2 Komagome, Toshima, Tokyo 170-0003, Japan \\ ${ }^{4}$ Nephrology, Dokkyo Medical University, Koshigaya Hospital. 2-1-50 Minamikosigaya, \\ Koshigaya, Saitama 343-0845, Japan \\ ${ }^{5}$ Social Medicene, Tokyo University of Pharmacy and Life Science. 1432-1 Horinouchi, \\ Hachiohji, Tokyo 192-0355, Japan
}

Received 24 December, 2014/Accepted 1 October, 2016

\begin{abstract}
Aquatic bacteria were isolated from the hands of working staffs by an adapted culture protocol. When the sample solution obtained by the "glove juice method" was incubated for 3 days at room temperature, viable cell counts increased up to $10^{5}$-fold, and the majority of the isolated colonies were shown to be Gram-negative aquatic bacteria, which carry the risk of contaminating water. Using R2A medium, coagulase-negative staphylococci were the dominant microbes immediately after recovery from the hands. Here it was revealed that bacteria of the phylum Proteobacteria isolated from the hand can be the causative bacteria of aqueous contamination. This modification in the GJ method may be useful as an effective training protocol to demonstrate the importance of hand hygiene and clean operation for aseptic manufacturing.
\end{abstract}

Key words : Dialysate / Endotoxin / Hemodialysis / Touch contamination / Water pollution.

In aseptic manufacturing products, the prevention of microbial contamination from humans is of the highest importance. As in the food and pharmaceuticals, preparation of dialysate for use in hemodialysis therapy must be kept sanitary, as well as sterile (ISO 2014, Kawanishi et al. 2009), due to its contact with blood through a semi-permeable membrane. Previously, we reported that the incidence of dialysate contamination increased, as shown by an increase in endotoxin value, when maintenance of the central dialysate delivery system (CDDS) was performed without hand hygiene (Honda et al. 2010). In DNA sequencing analysis of skin microbes, independent of culture method, the main bacteria of the hands have been reported to be Gramnegative aquatic bacteria, such as Proteobacteria (Fierer

*Corresponding author. Tel: +81-48-978-1611, Fax: +81-48978-1641, E-mail : e.osono(a)nms.ac.jp et al. 2008, Grice et al. 2009, Kong 2011), which have been shown to be able to contaminate dialysate (Oie et al. 2003). However, when bacteria from the hands were isolated and cultured, microbes from the phylum Firmicutes, such as coagulase-negative staphylococci (CNS), predominate despite using a medium suitable for aquatic bacterial culture (Cook 2007).

We attempted to isolate the aquatic bacteria inhabiting the hands by bacterial culture to demonstrate that hand hygiene associated with contamination of waterbased materials, such as dialysate. Sampling was conducted on 11 dialysis room staff members (doctors, nurses, and clinical engineers) after treatment or operation in the dialysis room. To collect bacteria, the dominant hand was washed in a 100-ml sterile water in a sterile bag (glove juice method (GJ) by Kampf et al. 2006). The GJ method was done using sterile saline for six samples (Fuso Pharmaceutical Industries, Ltd., 
Osaka, Japan), by the original method, or using dialysate treated by $0.22-\mu \mathrm{m}$ microfiltration (Ringer's solution containing $100 \mathrm{mg} / \mathrm{dl}$ glucose and divalent ion $\mathrm{Ca}$ in sodium bicarbonate buffer ( $\mathrm{pH}$ 7.4) prepared by the CDDS method (approved by ISO23500, ISO 2014)) for eight samples. The non-dominant hands were also tested on a different day (Fierer et al. 2008) for three staff members. Bacteria on the hands were investigated on the collected sample solutions immediately after sampling, defined as "at GJ", as well as collected sample solutions that were sealed in a bag and incu- bated for three days at room temperature in the dark, defined as "incubation" samples.

The samples were diluted with sterile distilled water for preparation of a 10-fold dilution series for viable cell counting on R2A medium (Merck Millipore Japan, Tokyo), which is suitable for culturing aquatic and heterotrophic bacteria in dialysate (ISO 2014, Kawanishi et al. 2009). The dilution series $(0.1 \mathrm{ml}$ each) were inoculated to the medium in two dishes, followed by a 5 -day incubation period at $25^{\circ} \mathrm{C}$. Viable cell counts were determined using the dilution rate of the plate containing a)

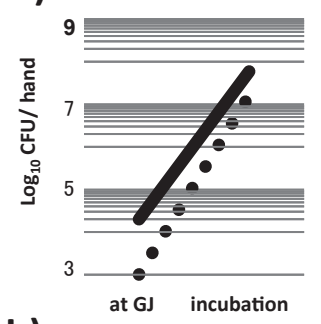

b)

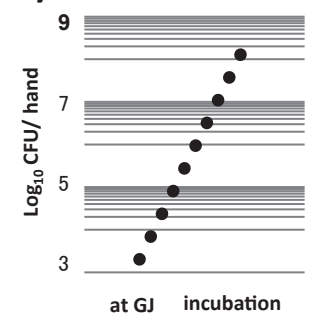

c)
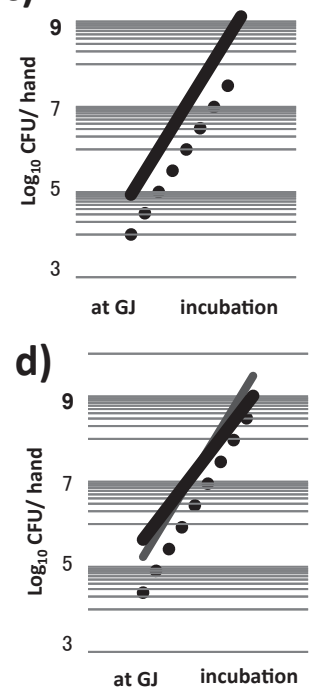

e)

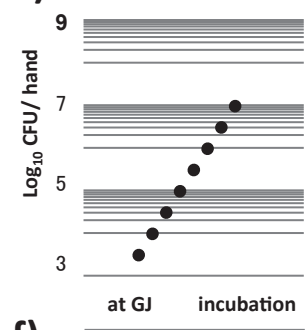

f)

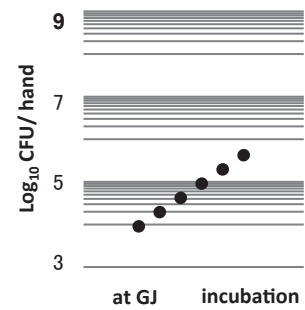

g)
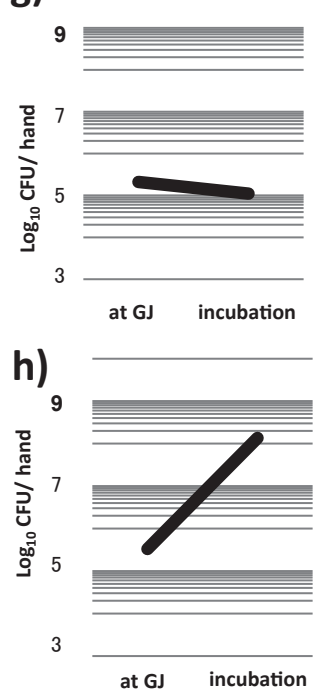
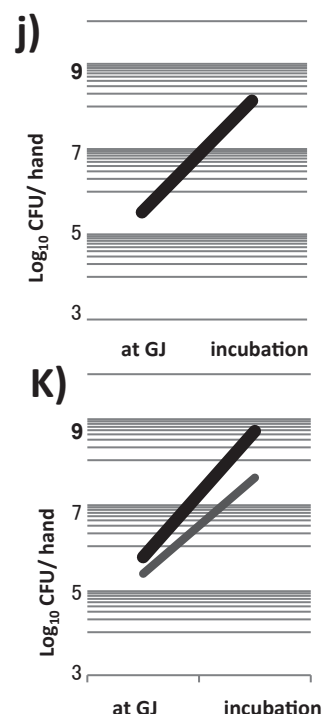

I)

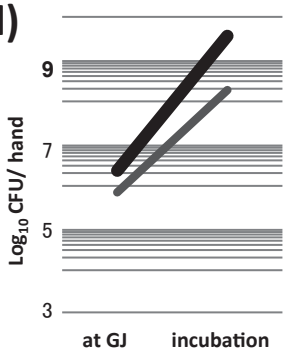

FIG. 1. Changes in viable cell counts at immediately after GJ and after incubation.

Viable cell counts were based on a 10-fold dilution series of the plate containing a range of 30-300 CFU and the actual number of colonies. The dashed lines shows the samples obtained in saline, and the solid line shows those in dialysates; the thin line shows samples from the non-dominant hand. 
a range of 30-300 CFU to calculate the actual number of colonies. Colonies were categorized according to their shape, color, and gram staining, and individual colony counts were recorded to reflect the ratios of bacterial species; differences within a dilution series was identified as logarithmic, and differences within the same dilution plate was linear. After 2 to 3 passages of the isolated colonies on R2A medium, purity was checked by isolating bacterial DNA (QIAMP DNA mini kit, Qiagen, Tokyo, Japan) and amplifying by PCR using universal primers for $16 \mathrm{~S}$ rRNA gene analysis (E. coli 8F and 1510r, Weisburg et al. 1991). The obtained bands were purified (Takara easy filtration kit, Takara, Osaka, Japan), and sequenced using an automatic analyzer (Applied Biosystems 3730 DNA analyzer, AB, Life Technologies Japan, Tokyo) with the 5'-side 8F primer. Bacterial species were identified based on the homology of the nucleotide sequences between nucleotides 8-825 using DDBJ BLAST and NCBI GenBank Blast. Of the candidate species, those with characteristics during culture that corresponded most to the descriptions of bacterial species and genera in Bergey's Manual of systemic Bacteriology $2^{\text {nd }}$ edition were selected. Statistical processing was performed with EZR software.

All results are shown in Figure 1. Bacteria were isolated from all collected solutions. The number of colonies from the "at GJ" samples were $10^{3}-10^{5} \mathrm{CFU} /$ one hand for the saline samples (dashed line) and $10^{4}-10^{7} \mathrm{CFU} /$ one hand for the dialysate samples (solid line). After a 3 day-incubation of the solutions at room temperature, the number of bacteria significantly increased 10 to $10^{5}$-fold (Students' paired $t$-test, $P<0.0001$ ). The bacteria from both the "at GJ" and "incubation" samples were divided into 1-4 categories based on bacterial shape. All bacteria were able to be grown in pure culture using R2A medium. A total of 81 colonies were isolated. The microscopic images of Gram-stained samples demonstrated that the majority of the colonies present in the "at GJ" samples were Gram-positive cocci: $96.5 \%$ in saline and $85.6 \%$ in dialysate (Figure 2a). Gram-negative cocci, Gram-positive bacilli, and Gram-negative bacilli comprised 0 to $7.6 \%$ of the a)
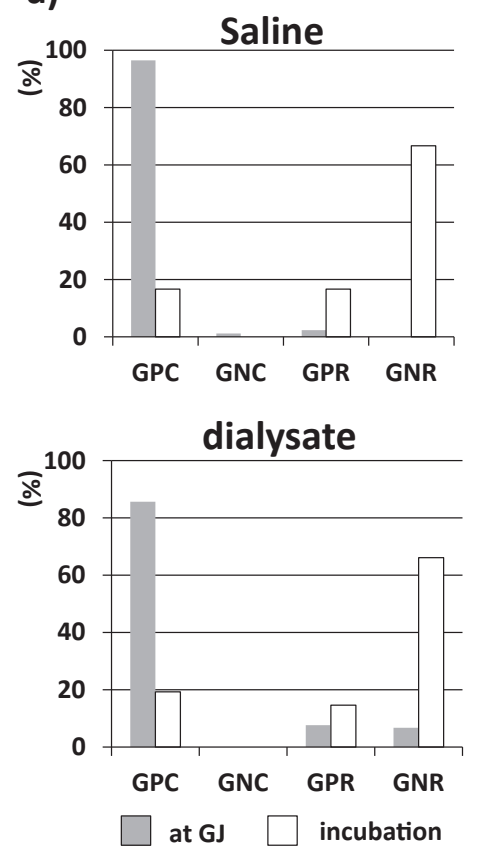

b)

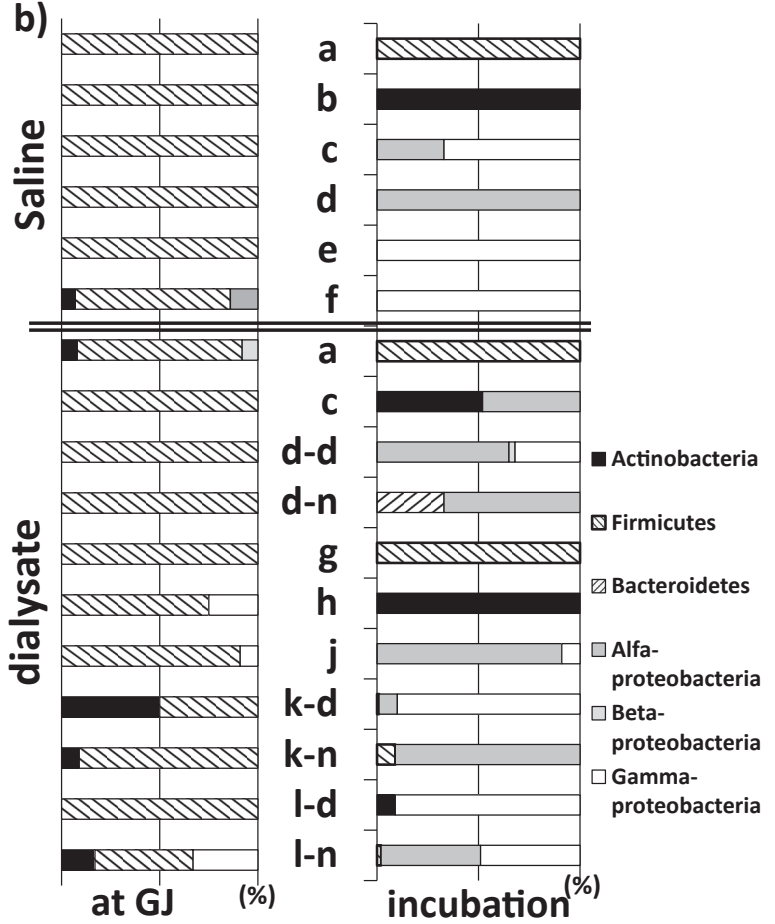

FIG. 2. Proportions and distributions of isolated bacteria species cultured from "at GJ" and "incubation" samples.

a): Gram staining. The proportion of bacterial species was significantly different between the "at GJ" and "incubation" samples in both saline (upper, $\mathrm{n}=6$ ) and dialysate (lower, $\mathrm{n}=$ 11, $\chi$-square test, $P<0.0001)$. Here, GPC, GPR, GNC, and GNR mean gram-positive coccus gram-positive bacillus (rod), and gram-negative cocci and gram-negative bacillus (rod), respectively.

b): Distribution of Phyla by $16 S$ rRNA genome analysis of each sample. Distributions are significantly different between the "at GJ" and "incubation" samples in both saline and dialysate (Predominance of phylum Firmicutes by Fisher's exact probability, $P<0.0001$ ). 
TABLE 1. Changes in bacterial strains between immediately after GJ and after incubation.

\begin{tabular}{|c|c|c|c|c|c|c|c|c|c|}
\hline \multicolumn{3}{|c|}{ at GJ } & \multicolumn{2}{|l|}{ incubation } & \multicolumn{3}{|c|}{ at GJ } & \multicolumn{2}{|l|}{ incubation } \\
\hline \multirow{8}{*}{ a) $\begin{array}{l}\text { S } \\
S \\
S \\
D \\
R\end{array}$} & Dialysate & & & & f) & Saline & & & \\
\hline & Staphylococcus pasteuri & 10 & Bacillus subtilis & & & Staphylococcus pasteuri & 10 & Enterobacter sp. & 10 \\
\hline & Staphylococcus warneri & 10 & & & & Paracoccus sp. & 2 & Erwinia tasmaniensis & 1 \\
\hline & Delftia lacustris & 1 & & & & Corynebacterium & & & \\
\hline & Rothia amarae & 1 & & & & tuberculostearicum & 1 & & \\
\hline & Saline & & & & g) & Dialysate & & & \\
\hline & Staphylococcus capitis & & Staphylococcus pasteuri & 2 & & Staphylococcus haemolyticus & 1 & Staphylococcus pasteuri & \\
\hline & & & Staphylococcus epidermidis & 1 & & Staphylococcus pasteuri & 1 & & \\
\hline & Saline & & & & h) & Dialysate & & & \\
\hline & Staphylococcus epidermidis & & Corynebacterium propinquum & & & Staphylococcus haemolyticus & 3 & Rothia amarae & \\
\hline \multirow{7}{*}{ C) } & Dialysate & & & & & Klebshiella sp. & 1 & & \\
\hline & Staphylococcus epidermidis & 2 & Kocuria kristinae & 10 & j) & Dialysate & & & \\
\hline & Staphylococcus haemolyticus & 1 & Brevendimonas diminuta & 10 & & Staphylococcus pasteuri & 10 & Agrobacterium larrymoore & 10 \\
\hline & & & Arthrobacter aurescens & 1 & & Acinetobacter junii & 1 & Acinetobacter junii & 1 \\
\hline & Saline & & & & K) & Dialysate & & & \\
\hline & Staphylococcus haemolyticus & & Pseudomonas oleovorans & 2 & & Kocuria rosea & 10 & Acinetobacter junii & 100 \\
\hline & & & Brevendimonas diminuta & 1 & & Staphylococcus pasteuri & 10 & Sphingomonas melonis & 10 \\
\hline \multirow{5}{*}{$\begin{array}{l}\text { d) } \\
S \\
S\end{array}$} & Dialysate & & & & & Kocuria kristinae & 1 & Staphylococcus pasteuri & 1 \\
\hline & Staphylococcus epidermidis & 1 & Brevendimonas nasdae & 20 & & Staphylococcus epidermidis & 1 & & \\
\hline & Staphylococcus pasteuri & 1 & Pseudomonas oleovorans & 10 & & Dialysate (non-dominant) & & & \\
\hline & & & Massilia timonae & 1 & & Staphylococcus pasteuri & 10 & Novosphingobium & \\
\hline & & & Methylobacterium sp. & 1 & & Kocuria rosea & 1 & subarcticum & 10 \\
\hline \multirow{9}{*}{ e) } & Dialysate (non-dominant) & & & & & & & Staphylococcus epidermidis & 1 \\
\hline & Staphylococcus epidermidis & 1 & Brevendimonas sp. & 2 & l) & Dialysate & & & \\
\hline & Staphylococcus pasteuri & 1 & Bacteroidetes endosymbiont & 1 & & Staphylococcus pasteuri & 2 & Acinetobacter junii & 100 \\
\hline & saline & & & & & Staphylococcus haemolyticus & 1 & Microbacterium imperiale & 1 \\
\hline & Staphylococcus pasteuri & & Brevendimonas nasdae & 1 & & Dialysate (non-dominant) & & & \\
\hline & & & Methylobacterium sp. & 1 & & Staphylococcus pasteuri & 3 & Nobosphingobium & \\
\hline & Saline & & & & & Kocuria rosea & 2 & subarcticum & 100 \\
\hline & Staphylococcus capitis & & Pantoea dispersa & 3 & & Escherichia coli & 1 & Acinetobacter septicus & 100 \\
\hline & & & Enterobacter sp. & 1 & & & & Staphylococcus pasteuri & 1 \\
\hline
\end{tabular}

Bacterial species and strains were identified by $16 \mathrm{~S}$ rRNA gene homology. The numbers noted after the strains are based on colony counts that were recorded to reflect the ratios between bacterial species: differences within a dilution series are denoted as 1 , 10, and 100 times higher, and differences within the same dilution plate as 1, 2, and 3 times higher.

samples. However, the "incubation" samples showed a decrease in Gram-positive cocci (16.7\% in saline and $19.3 \%$ in dialysate), while Gram-negative bacilli increased to $66.7 \%$ in saline and $66.1 \%$ in dialysate ( $X$-square test, $P<0.0001$ ). Gram-positive bacilli also increased from $2.3 \%$ to $16.7 \%$ in saline, and from 7.6 to $14.6 \%$ in dialysate. These changes in composition, resulting from the increased bacilli and decreased Gram-positive cocci were shown to be significant by Bonferroni's post hoc test.
By 165 rRNA genome analysis, the genotypes of 70 strains (excluding overlapping strains in the same sample) were identified. The predominant phylotype of the "at GJ" samples was the phylum Firmicutes (Figure 2b). Coagulase-negative staphylococci, such as Staphylococcus epidermidis and S pasteuri, were isolated from all 17 "at GJ" samples (Table 1). Both Proteobacteria and Actinobacteria were isolated in five samples, although they were the minority of microbes. The "incubation" samples showed a marked change in 
microbe distribution, specifically, the bacteria of the phylum Firmicutes were predominant in only three samples (17.6\%). Actinobacteria, alpha-Proteobacteria and gamma-Proteobacteria were predominant in 14 samples (82.4\%, Fisher's exact probability, $P=0.011$, Figure $2 \mathrm{~b}$ ). These results suggested that contamination by Gram-negative bacilli, a cause of endotoxin activity, may be derived from the hands (Figure 2). While coagulase-negative staphylococci were still isolated from five "incubation" samples (case a, g, k dominant, k nondominant, and I in table), these were outnumbered by the increase in other microbes both in saline and dialysate. Similar results were observed when observing both the dominant and non-dominant hands of the same person (case $\mathrm{d}, \mathrm{k}$, and $\mathrm{l}$ ), in confirmation with an early report (Fierer et al. 2008). After incubation, neither the bacterial growth rates nor the microbe composition were different between saline and dialysate (case a, c, and $d$ in Figures 1 and table). The "at GJ" samples showed a higher diversity and harvested cell count in the dialysate, which may have been due to the presence of sugar or $\mathrm{Ca}^{2+}$ (average 2.45 stains in all dialysate and 1.33 in all saline. $P=0.017$ by Students' unpaired $t$-test).

In conclusion, it is shown that bacteria of the phylum Proteobacteria, are the causative bacteria of aqueous contamination, because they were isolated from the hands of working staffs. Our modification in the GJ method was simple and significant in revealing the bacteria attached to the hands as a source of aqueous contamination. This method is reproducible with the specified medium, formulation of interest, and Gram stain, which may be used as an effective training protocol without need of any specialized equipment for hand hygiene procedures.

\section{REFERENCES}

Cook, H. A., Cimiotti, J. P., Della-Latta, P., Saiman, L., Larson, E. L. (2007) Antimicrobial resistance patterns of colonizing flora on nurses' hands in the neonatal intensive care unit. Am. J. Infect. Control., 35, 231-236.

Fierer, N., Hamady, M., Lauber, C. L., Knight, R. (2008) The influence of sex, handedness, and wand washing on the diversity of hand surface bacteria. ProNAS, 105, 1799417999.

Grice, E. A., Kong, H. H., Conlan, S., Deming, C. B., Davis, J., Young, A. C.; NISC Comparative Sequencing Program, Bouffard, G. G., Blakesley, R. W., Murray, P. R., Green, E. D., Turner, M. L., and Segre, J. A. (2009) Topographical and temporal diversity of the human skin microbiome. Science, 324, 1190-1192.

Honda, K., Inoue, Y., Osono, E., Ichimura, K., Norose, Y., Takahashi, H., and Hayama, N. (2010) Hand hygiene is essential for avoiding bacterial contamination during hospital preparation of dialysis fluid. Jpn. J. Soc. Dial. Ther., 43, 361366.

ISO (2014). Guidance for the preparation and quality management of fluids for hemodialysis and related therapies. ISO 23500:2014.

Kampf, G., Ostermeyer, C., Heeg, P., and Paulson, D. (2006) Evaluation of Two Methods of Determining the Efficacies of Two Alcohol-Based Hand Rubs for Surgical Hand Antisepsis. Appl. Environ. Microbiol., 72, 3856-3861.

Kawanishi, H., Akiba, T., Masakane, I., Tomo, T., Mineshima, M., Kawasaki, T., Hirakata, H., and Akizawa, T. Standard on Microbiological Management of Fluids for Hemodialysis and Related Therapies by the Japanese Society for Dialysis Therapy. (2009) Ther. Apher. Dial., 13, 161-166, 2009.

Kong, H. H. (2011) Skin microbiome: genomics-based insights into the diversity and role of skin microbes. Trends Mol. Med., 17, 320-328.

Oie, S., Kamiya, A., Yoneda, I., Uchiyama, K., Tsuchida, M., Takai, K., and Naito, K. (2003) Microbial contamination of dialysate and its prevention in haemodialysis units. J. Hosp. Infect., 54, 115-119.

Weisburg, W. G., Barns, S. M., Pelletier, D. A., Lane, D. J. (1991) 16S ribosomal DNA amplification for phylogenetic study. J. Bacteriol., 173, 697-703. 